\title{
Aktuelle Behandlungsstrategien von knöchernen Verletzungen der Orbitae
}

$\square$ Claude Jaquiéry, Christoph Leiggener, Carl-Peter Cornelius, Christoph Kunz

\section{Zusammenfassung}

Frakturen des Jochbeinkomplexes und der Orbita sind häufig. Stumpfe Traumata (Schlag, Sturz) führen zu einer Impression der Jochbeinprominenz mit Frakturen an anatomisch vorgegebenen Schwachstellen. Eine Beteiligung des Orbitabodens ist obligat. Ein relevanter Defekt des Orbitabodens kann u.U. erst nach der Reposition des Jochbeinkomplexes sichtbar werden, weshalb eine Exploration des Orbitabodens nach Reposition und Osteosynthese erfolgen sollte, sofern keine intraoperative Bildgebung zur Kontrolle zur Verfügung steht. Hochgeschwindigkeitstraumata führen zu komplexen Mittelgesichtsverletzungen und irregulären Frakturmustern. Die CT-Diagnostik des Mittelgesichts und der Orbitae hat die konventionelle Bildgebung verdrängt. Knöcherne Verletzungen der Orbitae können initial ohne wesentliche klinische Symptomatik einhergehen, weshalb erst in der Feinschichtcomputertomografie eine Fraktur der Orbita erkannt werden kann. Die Indikation zur operativen Versorgung einer Fraktur wird nach Berücksichtigung von klinischen und radiologischen Befunden gestellt. Doppelbildsehen ist ein wichtiges klinisches Kriterium für die Operation, kann aber nur von temporärer Dauer sein. Neben der Ausdehnung der Defektfraktur im CT $\left(\geq 3 \mathrm{~cm}^{2}\right)$ ist ein Enophthalmus ( $\geq 2 \mathrm{~mm}$ ) der wichtigste klinische Parameter für die Entschei- dung zur operativen Revision. Für die Rekonstruktion der Orbitawände werden heute mehrheitlich Titangitter mit spezifischem Design eingesetzt. In bestimmten klinischen Situationen (Rekonstruktion nach Tumorresektion) sind autologe Transplantate (tabula externa) immer noch zu favorisieren. Bezüglich Präzision und postoperativer Komplikationsrate bestehen keine Unterschiede zwischen autologen Transplantaten und Titanimplantaten. Die 3dimensionale Planung und intraoperative Navigation hat sich v.a. bei den sekundären Korrekturen von in Fehlstellung verheilten Mittelgesichtsverletzungen bewährt. Die funktionellen Resultate nach sekundärer Korrektur eines Enophthalmus sind nicht ermutigend. Eine besondere Gewichtung erhält deshalb die präzise klinische und radiologische Diagnostik, die korrekte Indikationsstellung und die primäre sowie zeitnahe Versorgung von knöchernen Verletzungen der Orbita.

\section{Treatment of Orbital Wall Fractures: Current Concepts}

Fractures of the zygomatic complex and the orbit are common. They occur in general after blunt trauma (fall, blow) leading to impression of the zygomatic complex due to fractures of relatively weak anatomic sutures involving the orbital floor. However, comminuted fractures of the midface are the result of high energy trauma. Orbital wall de- fects might be visible only after repair of the zygomatic complex. Hence, exploration of the orbital floor should be performed after stabilisation of zygomatic fractures. If available, intraoperative CT could exclude operatively relevant defects of orbital walls. Initially, fractures of the orbit might occur without relevant clinical signs (double images, enophthalmos). ACT scan is therefore mandatory to achieve a correct diagnosis. Surgical repair follows thorough clinical and radiological assessment, only. Double images are relevant clinical signs, but might be of temporal character. The size of the fracture measured on CT scans $\left(\geq 3 \mathrm{~cm}^{2}\right)$ and enophthalmos ( $\geq 2 \mathrm{~mm}$ ) are most important parameters supporting surgical intervention. Nowadays, reconstruction of orbital walls is performed by using specifically designed titanium meshes. For distinct clinical indications (resection of tumours) autologous bone (tabula externa) is still to be preferred. In terms of precision and postoperative complication rate, no relevant differences can be found. Three-dimensional planning and intraoperative navigation are being more and more used in the context of secondary corrections. However, the results after secondary corrections of enophthalmos are not encouraging. In order to achieve best results, precise clinical and radiological diagnostics including a correct indication followed by early and anatomic repair of orbital wall fractures should be performed.

\section{Einleitung}

Die Orbita ist eine komplexe anatomische Struktur und setzt sich aus mul-

OP-JOURNAL 2013; 29: 136-146

(c) Georg Thieme Verlag KG Stuttgart · New York DOI http://dx.doi.org/10.1055/s-0033-1350790 tiplen Anteilen von Gesichtsschädelknochen (Abb. 1 a) zusammen. Im anterioren Bereich erscheint ein koronarer Schnitt durch die Orbita quadratisch (Abb. 1b), wohingegen im dorsalen Abschnitt eher ein dreieckförmiger Querschnitt beschrieben werden kann. Diese anatomischen Besonderheiten müssen im Rahmen der Rekonstruktion der Orbi- tawände berücksichtigt werden. Der Inhalt der Orbita besteht neben dem Bulbus aus periorbitalem Gewebe, worin die Augenmuskeln, Nerven und Gefäße, sowie Drüsengewebe eingebettet sind (Abb. 1 c1-c6). Innerhalb dieses periorbitalen Gewebes sind die Augenmuskeln durch feine bindegewebige Septen miteinander verbunden. Dieses komplizier- 


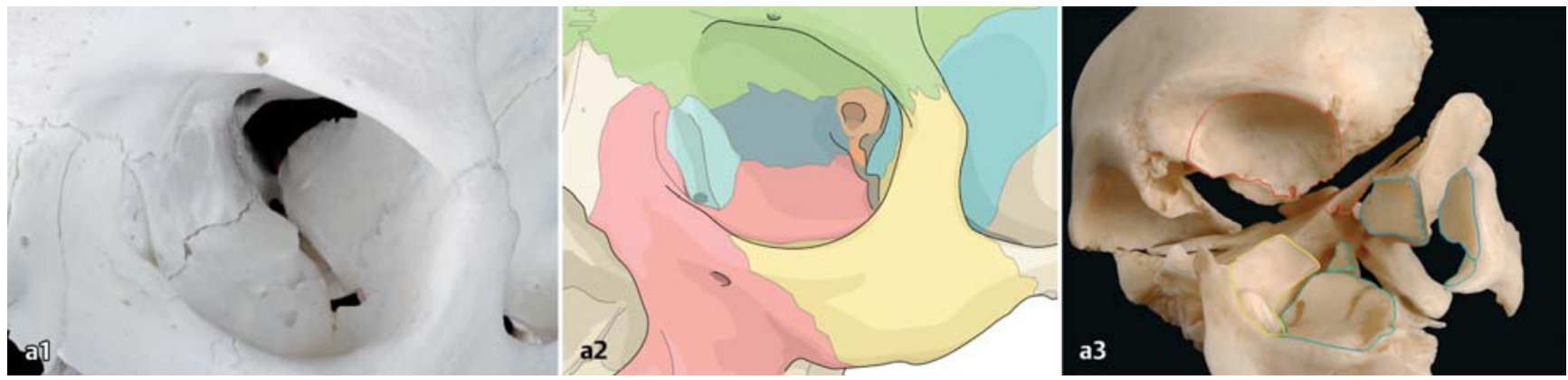

Abb. 1 a a1 Orbita links. a2 Orbita links: oliv-grün: os frontale, violett: maxilla, gelb: os zygomaticum, türkis: os lacrimale (medial), blau: os sphenoidale, graublau: os ethmoidale, lamina orbitalis. a3 Orbita links - Einzelknochen gesprengt und geöffnet - Komponenten der internen Orbita bzw. Orbitawände (farbig umrandet): grün: Orbitaboden, gelb: mediale Orbitawand, hellblau: laterale Orbitawand, rot: Orbitadach.

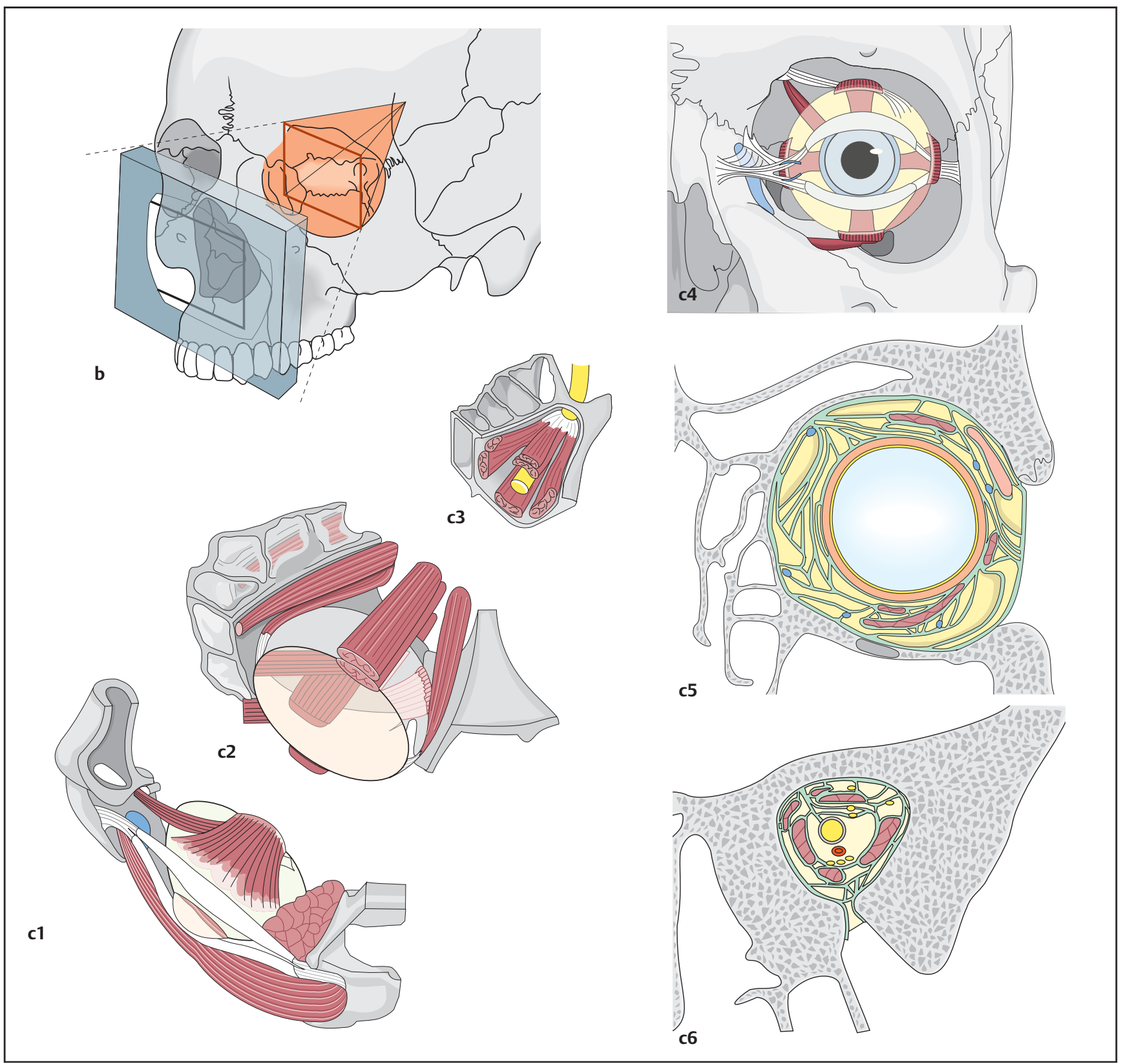

Abb. $\mathbf{1}$ b und c b Konzeption der Orbita als Pyramide mit quadratischer Basis. Die Orbitaränder bilden den äußeren Rahmen, die Orbitawände die Pyramidenseite. Da der Orbitaboden kürzer ist als die anderen Wände, nimmt die Pyramide an der Spitze eine Dreieckform an. c Übersicht über den Orbitainhalt in 3 Abschnitten: anterior, Midorbita, posterior und zugehörige Querschnitte. Im Querschnitt durch die Midorbita: quer angeschnittener Bulbus und quer angeschnittene Augenmuskeln, die in Fettgewebe eingebettet und von einem Netz aus feinen bindegewebigen Septen eingeschlossen sind, das sie untereinander verbindet und suspendiert. 


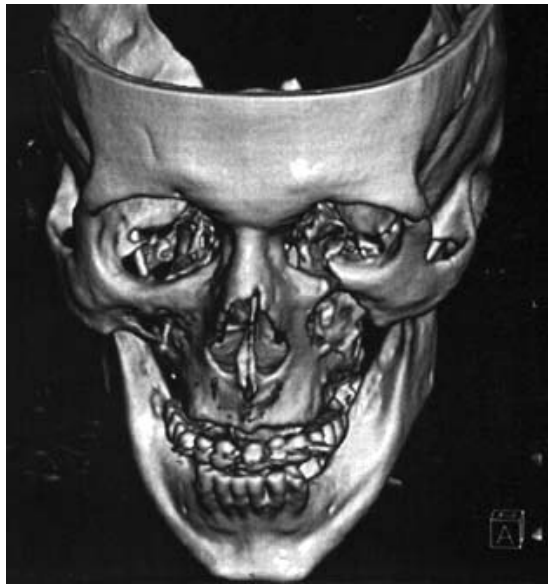

Abb. 2 Jochbeinfraktur links mit Impression der Jochbeinprominenz. Beachte: der Jochbogen ist nicht frakturiert, aber überbogen.

te System zur Suspension und gegenseitigen Stabilisierung der Augenmuskeln kann im Falle eines Traumas temporär oder bleibend gestört werden (Abb. 1 c5) [1].

Knöcherne Verletzungen der Orbitae werden in der Regel im Zusammenhang mit Frakturen des Jochbeinkomplexes diagnostiziert. Ausgangspunkt ist ein stumpfes Trauma auf die laterale Gesichtshälfte, das zu einer Impression der Jochbeinprominenz führt. Frakturen treten dabei auf (I) am Infraorbitalrand, (II) an der Crista zygomatico-alveolaris, (III) an der Crista fronto-zygomatica, (IV) am Jochbogenansatz mit Impression des Jochbogens $(V)$ sowie im Bereich des knöchernen Bodens der Orbita (Abb. 2). Isolierte Verletzungen der knöchernen Wände der Augenhöhle können durch direkte Krafteinwirkungen auf den widerstandsfähigen und elastischen Bulbus auftreten, der die Kraft auf die dünnen knöchernen Strukturen der medialen Wand und des Bodens überträgt. In diesem Zusammenhang spricht man von „Blow-out“ Frakturen (Abb.3a,b). Schließlich können komplexe knöcherne Verletzungen der Orbitae im Zusammenhang mit Mittelgesichtsfrakturen oder panfazialen Frakturen als Folge von Hochgeschwindigkeitstraumata beobachtet werden (Abb. 4) [2].

Pathophysiologisch kommt es bei Auftreten einer Defektfraktur im Bereich der Orbitawände zu einem Zurückfallen des periorbitalen Gewebes in den Defekt hinein (Enophthalmus), unter der Voraussetzung, dass die Fraktur dorsal des Bulbus-Äquators lokalisiert ist. Durch die Vergrößerung des orbitalen Volumens verändert sich die Vorspannung
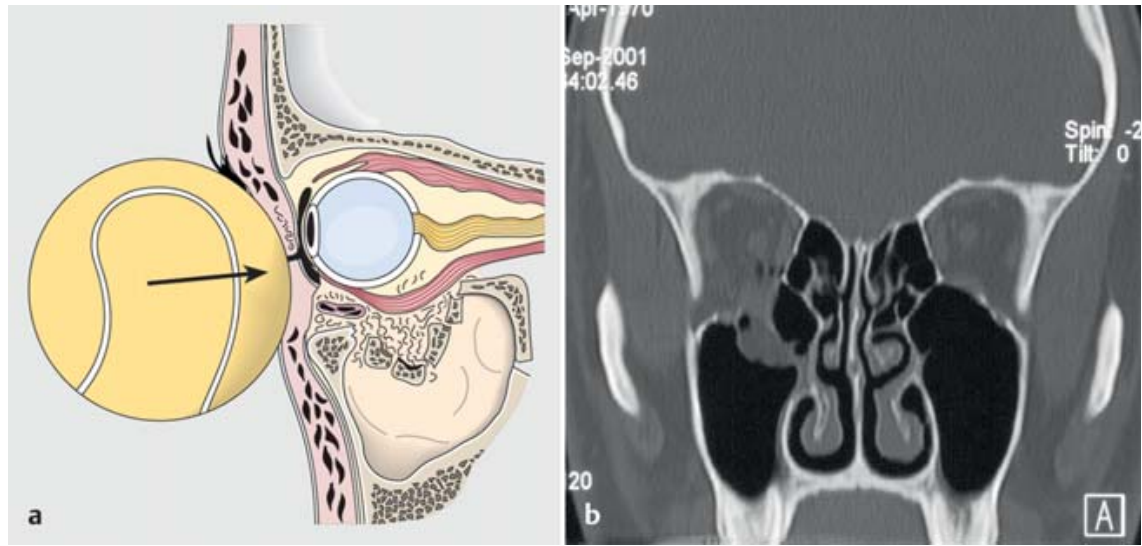

Abb. 3 a und b a Stumpfe Gewalt auf den Bulbus, Weiterleitung der Kraft auf den Orbitaboden, der frakturiert ist. b Aussprengung des Orbitabodens rechts im posterioren Anteil („Blow-out“Fraktur).

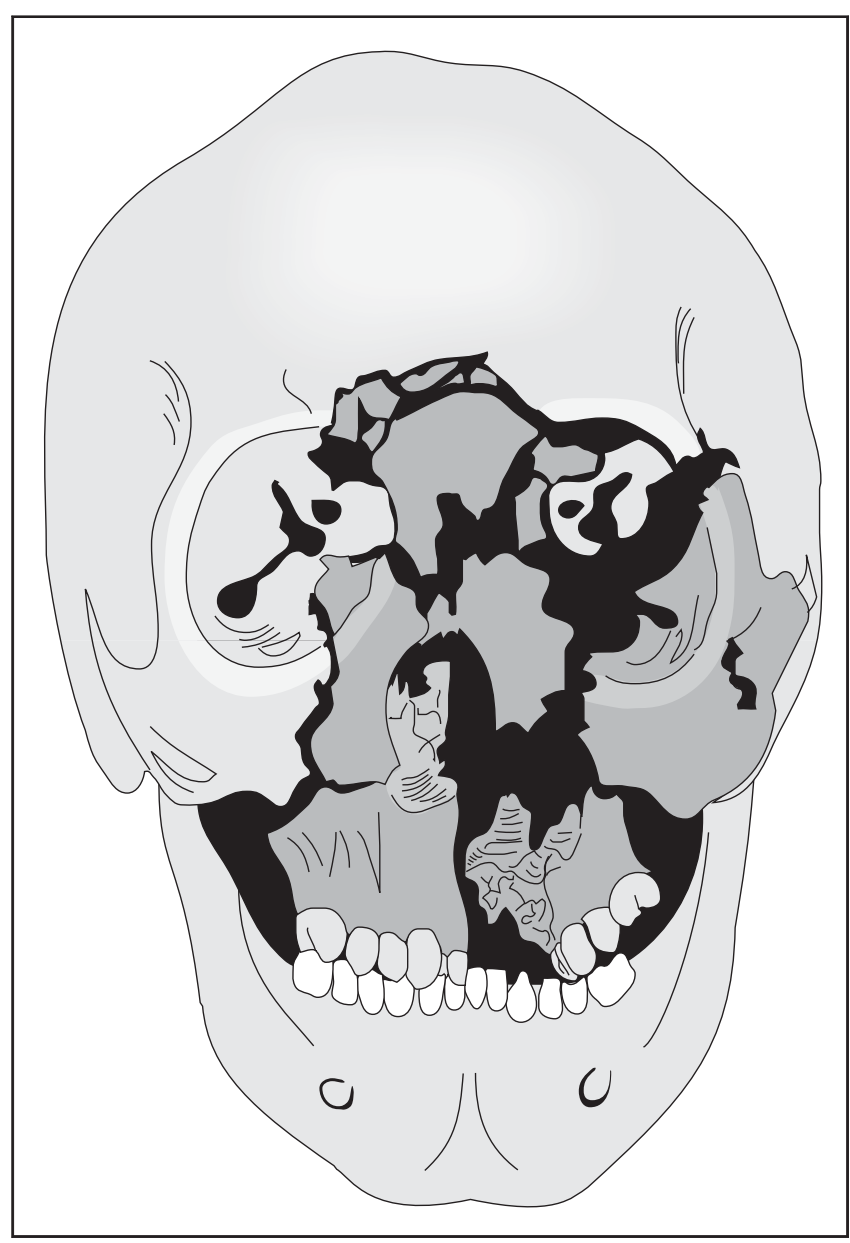

Abb. 4 Hochgeschwindigkeitstrauma des zentrolateralen Mittelgesichts mit irregulären und mehrfragmentären Frakturen.

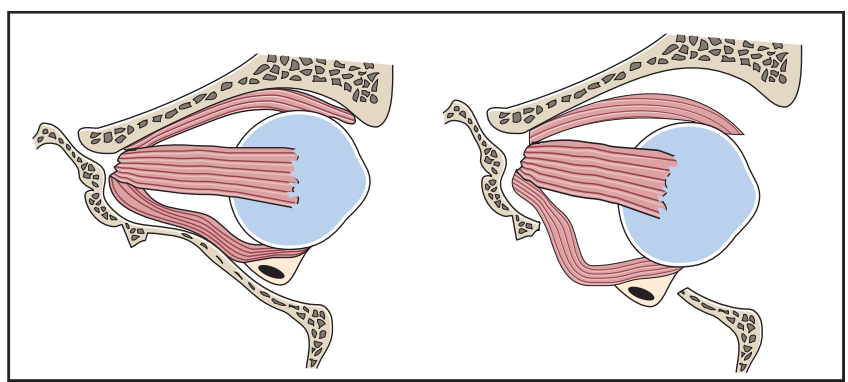

Abb. 5 Links: korrekte Position des Bulbus, rechts: Defekt des Orbitabodens, dadurch Zurückfallen des Bulbus (Enophthalmus) und Abnahme der Vorspannung der Augenmuskeln. 


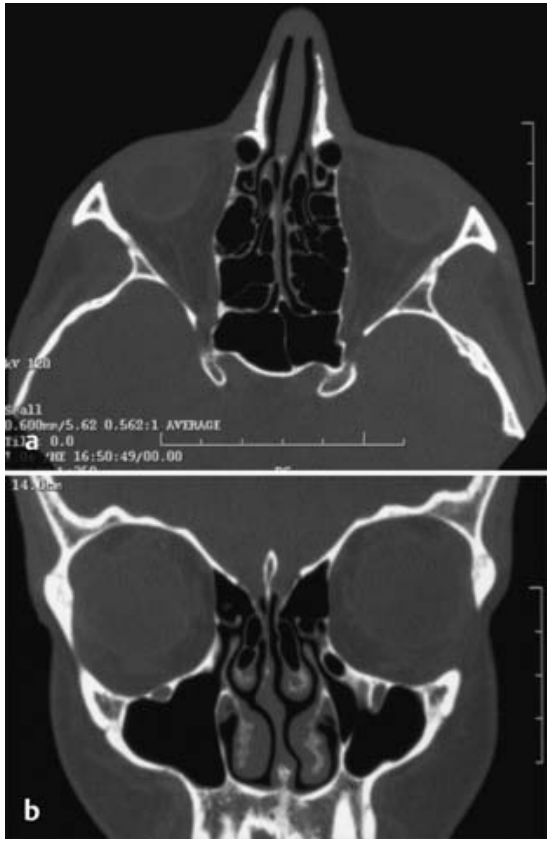

Abb. $6 \mathbf{a}$ und $\mathbf{b}$ a Feinschicht-CT der Orbita: axiale Schichtung. b Feinschicht-CT der Orbita: koronare Schichtung.

der Augenmuskeln, was zu einer gestörten Motilität und Koordination mit dem anderen Auge führen kann. In der Folge können Doppelbilder (Diplopie) in bestimmten Blickrichtungen oder sogar in der Primärstellung des Bulbus auftreten (Abb. 5)

\section{Diagnostik}

Die konventionelle Bildgebung wurde in den letzten Jahren durch die computertomografische (CT-) Diagnostik abgelöst. Da knöcherne Verletzungen der Orbitae ohne wesentliche klinische Symptome einhergehen können, ist die FeinschichtCT-Untersuchung die wichtigste diagnostische Maßnahme (Abb.6a,b). Bei Vorliegen von axialen und koronaren Schichtungen können sämtliche knöchernen Wände der Orbitae eingesehen und beurteilt werden. Klinisch muss, neben der Beurteilung der Sehschärfe, die Augenmotilität und die Projektion des betroffenen Auges im Vergleich zum gesunden Auge geprüft werden. Idealerweise wird die klinische Untersuchung durch den Ophthalmologen durchgeführt, der, bei guter Compliance des Patienten, eine orthoptische Beurteilung des Fusionsblickfelds vornehmen kann (Abb. 7a). Auf der sog. Harms-Tangententafel kann das Fusionsblickfeld des Patienten grafisch dargestellt werden. Die Projektion des betroffenen Auges im Vergleich zum gesunden Auge wird durch das Exophthalmometer nach Hertel oder Naugle (Abb. 7 b, c) bestimmt.

Dank des technologischen Fortschritts (verbesserte Auflösung, verminderte Strahlenbelastung, kurze Untersuchungszeiten) hat sich die Computertomografie als das Mittel der Wahl in der Diagnostik der Orbitafrakturen durchgesetzt und die konventionelle Bildgebung abgelöst.

\section{Indikation zur operativen Versorgung}

Im Zusammenhang mit der radiologischen Diagnostik sind die klinischen Parameter Doppelbildsehen und Enophthalmus die häufigsten Kriterien, die als Gründe für die operative Versorgung angegeben werden [3]. Ein rasch oder unmittelbar posttraumatisch aufgetretener Enophthalmus ist jedoch der wichtigste Indikator für eine notwendige knöcherne Rekonstruktion der Orbita. Doppelbildsehen und Motilitätsstörungen können, bedingt durch Schwellung und Hämatombildung, von temporärem Charakter sein. Unter verschiedenen Autoren besteht eine große Übereinstimmung darüber, dass die besten funktionellen Resultate durch eine möglichst frühe und anatomische Rekonstruktion der Orbita erreicht werden können [4,5]. Eine konservative Therapie ist bis zu einer Defektgröße von $3 \mathrm{~cm}^{2}$ möglich, unter der Voraussetzung, dass weniger oder gleich $2 \mathrm{~mm}$ Enophthalmus besteht und eine Einklemmung von Muskel- oder Weichgewebe ausgeschlossen werden kann [6].

Bei fehlendem Enophthalmus ( $\leq 2 \mathrm{~mm}$ ) können Orbitafrakturen bis zu einer Frakturfläche von $3 \mathrm{~cm}^{2}$ funktionell nachbehandelt werden. Doppelbilder sind meist von temporärem Charakter unter der Voraussetzung, dass eine Einklemmung von Weichgewebe in den Frakturspalt ausgeschlossen werden konnte. Frakturen, die anterior des Äquators des Bulbus liegen, sind funktionell nicht relevant und müssen nicht versorgt werden.
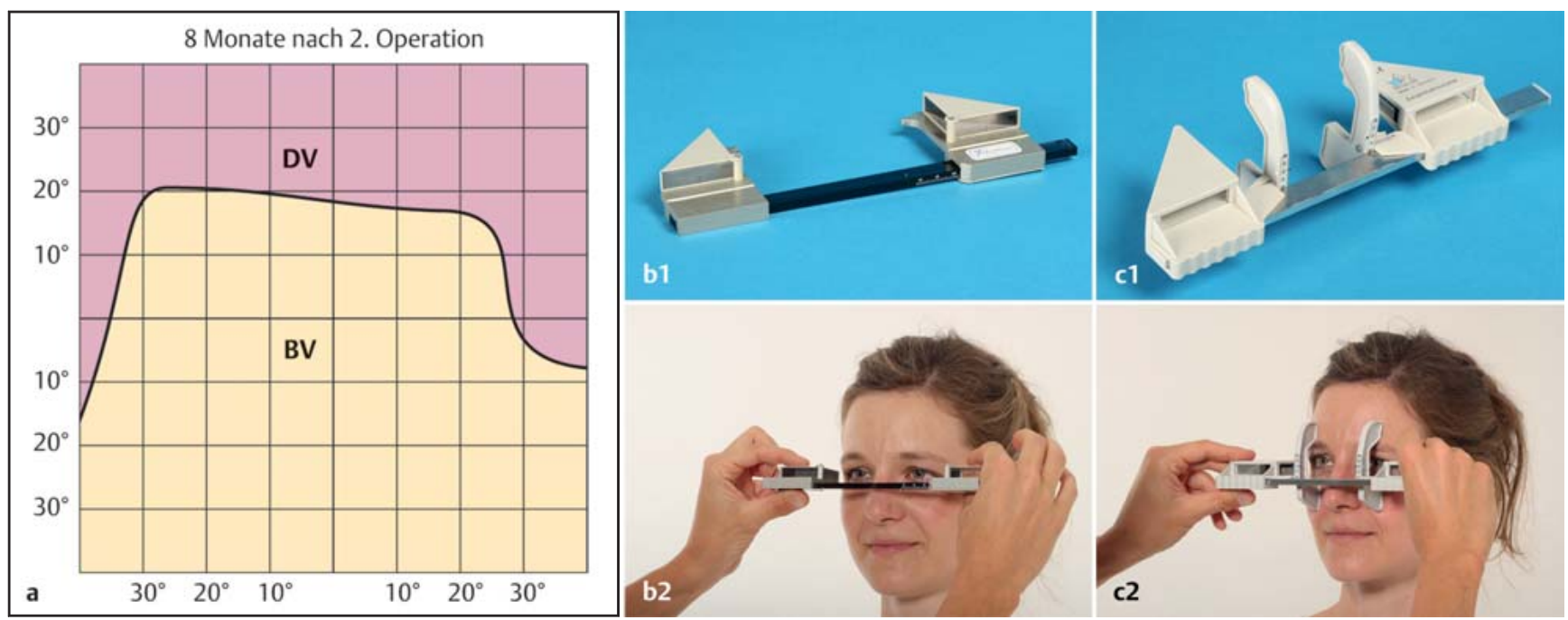

Abb. 7 a bis c a Harms-Tangententafel eines Patienten: violett: Doppelbildsehen nach oben ab $20^{\circ}$, seitlich nach $25^{\circ}$, resp. $30^{\circ}$, gelb: Fusionsblickfeld. b1 und b2 Hertl-Exophthalmometer: Über ein Spiegelsystem mit eingebauter Millimeterskala wird der Abstand vom lateralen Orbitarand zur Hornhaut gemessen, um so einen Enophthalmus oder Exophthalmus zu quantifizieren. Die Apparatur wird dabei auf dem seitlichen Orbitarand abgestützt (2-Punkt-Auflage), der nach Traumen asymmetrisch sein kann. c1 und c2 Naugl-Exophthalmometer. Die Naugl-Apparatur stützt sich jederseits auf dem Infra- und Supraorbitalrand ab (4-Punkt-Auflage). Neben sagittalen Abweichungen können auch vertikale Diskrepanzen (Hypoglobus/Hyperglobus) bestimmt werden.

Claude Jaquiéry et al.: Aktuelle Behandlungsstrategien von knöchernen Verletzungen der Orbitae 


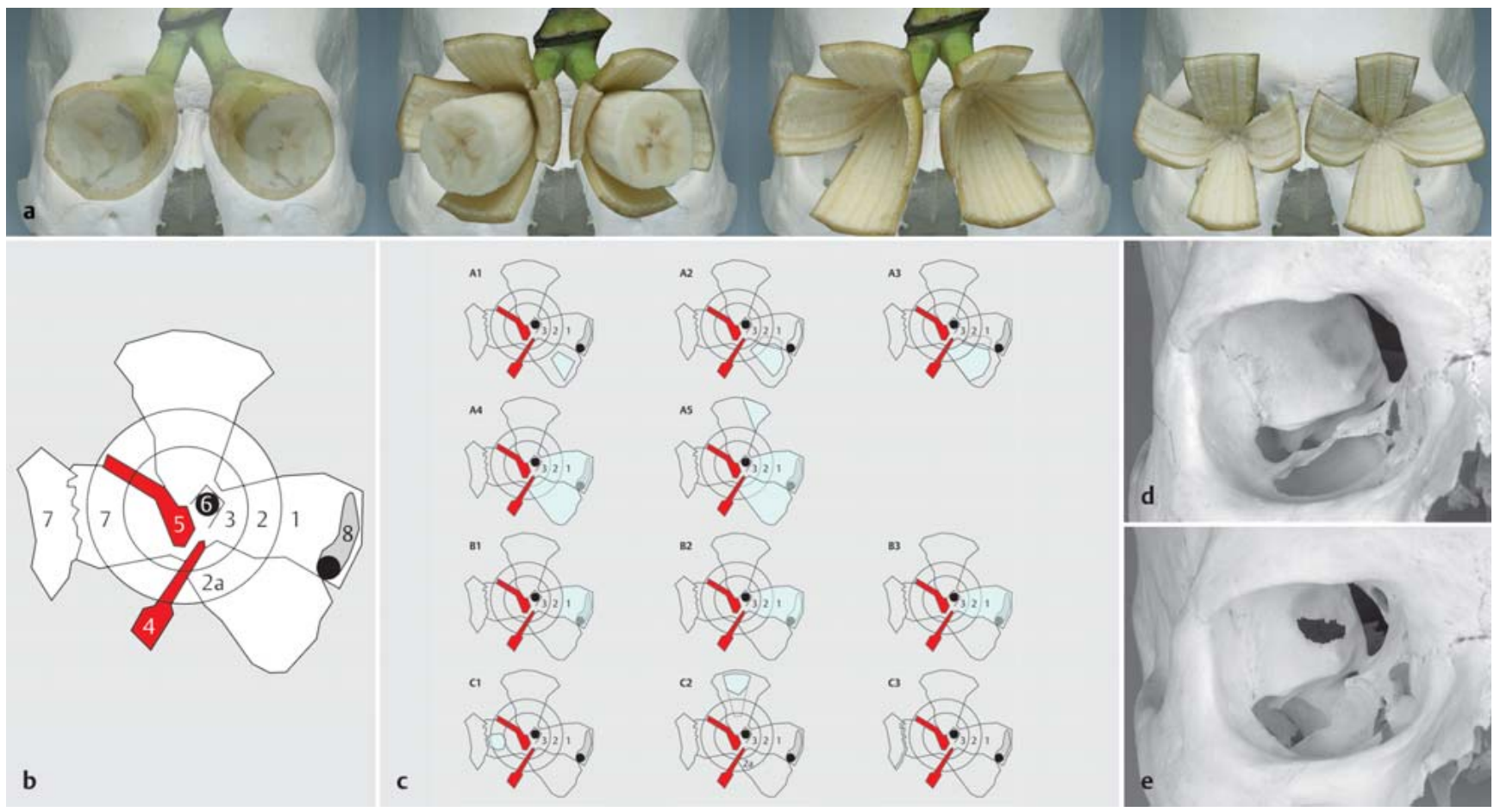

Abb. 8 a bis e a Durch Auffalten (wie beim Schälen einer Banane - Idee: C. P. Cornelius, Realisation: Klaus Völcker, Regensburg) der komplexen 3-dimensionalen Struktur der Orbita ergibt sich ein 2-dimensionales Schema. b Zweidimensionales Schema der Orbita rechts: 1: vorderes Drittel, 2: mittleres Drittel, 2 a: knöcherne Begrenzung der Fissura orbitalis inferior, 3: hinteres Drittel, 4: Fissura orbitalis inferior, 5: Fissura orbitalis superior, 6: N. opticus, 7: laterale Wand, 8: Tränensack. c Modifizierte Klassifikation der Orbitafrakturen nach Jaquiéry et al. A: Frakturen des Bodens, der medialen Wand und des Orbitadachs, B: isolierte Frakturen der medialen Wand, C: isolierte Frakturen der lateralen Wand und des Orbitadachs. d Fraktur des Orbitabodens der Kategorie A2: Die knöcherne Begrenzung der Fissura orbitalis inferior ist erhalten. e Fraktur des Orbitabodens der Kategorie A3: die knöcherne Begrenzung zur Fissura orbitalis inferior fehlt.

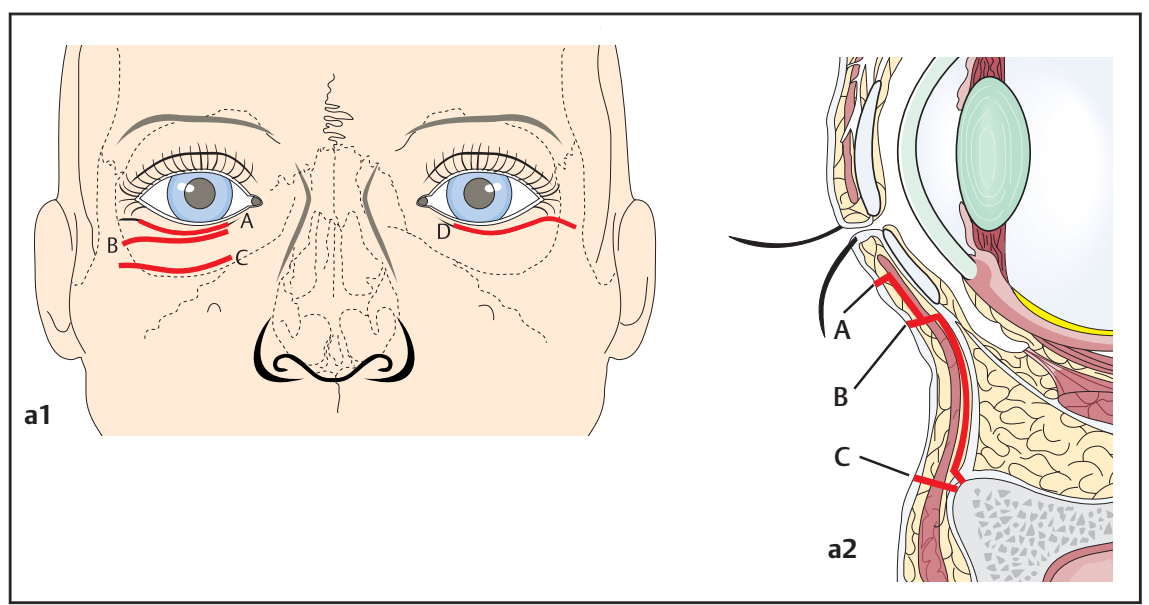

Abb. 9a a1 Lokale transkutane Unterlidzugänge zur Orbita im Schema: A subziliare Inzision; B subtarsale bzw. „Mideyelid-Incision“; C infraorbitale Inzision; D subziliare Inzision mit lateraler Erweiterung. a2 Lokale transkutane Unterlidzugänge zur Orbita in Sagittalschnitt-Ansicht. Die Präparation erfolgt immer vor dem Septum orbitale. Das sog. orbitale Septum ist eine Bindegewebsschicht dorsal des Ringmuskels (M. orbicularis oculi), der die Orbitaöffnung oberflächlich umgibt.

\section{Klassifikation von Frakturen der Orbitae}

Bei der Klassifikation von Frakturen der Orbitae müssen, neben der (i) Größe der Defekte auch die (ii) Lokalisation und (iii) definierte anatomische Landmarken mit einbezogen werden. Alle diese Parame- ter bestimmen die technischen Anforderungen im Rahmen der Rekonstruktion. Da die Orbita eine komplexe 3-dimensionale Struktur darstellt, ist die Einführung einer Klassifikation nicht ohne Simplifizierung respektive Überführung in ein 2-dimensionales Schema möglich. Gleich einer Banane können die Wände der Orbita aufgefaltet und in ein 2-dimensionales Modell übersetzt werden (Abb.8a). Durch die Bezeichnung von definierten anatomischen Strukturen kann jeder Defekt genau lokalisiert und im Schema eingetragen werden (Abb. 8b) [5]. Durch die Kombination eines Zahlen- und Buchstabencodes sind alle theoretisch möglichen Defekte klassifizierbar (Abb. 8c) [6]. Es konnte nachgewiesen werden, dass die funktionellen ophthalmologischen Langzeitbefunde dem Schweregrad einer bestimmten Kategorie entsprechen. Insbesondere zeigte sich, dass bei präoperativ intakter medialer knöcherner Begrenzung der Fissura orbitalis inferior bessere funktionelle Resultate erwartet werden können, als wenn präoperativ diese wichtige anatomische Struktur nicht mehr identifiziert werden kann (Abb.8d,e) [5]. Bei fehlender knöcherner Begrenzung der Fissura orbitalis inferior ist die Darstellung des Defekts, sowie die Rekonstruktion des Bodens der Augenhöhle technisch anspruchsvoller. 


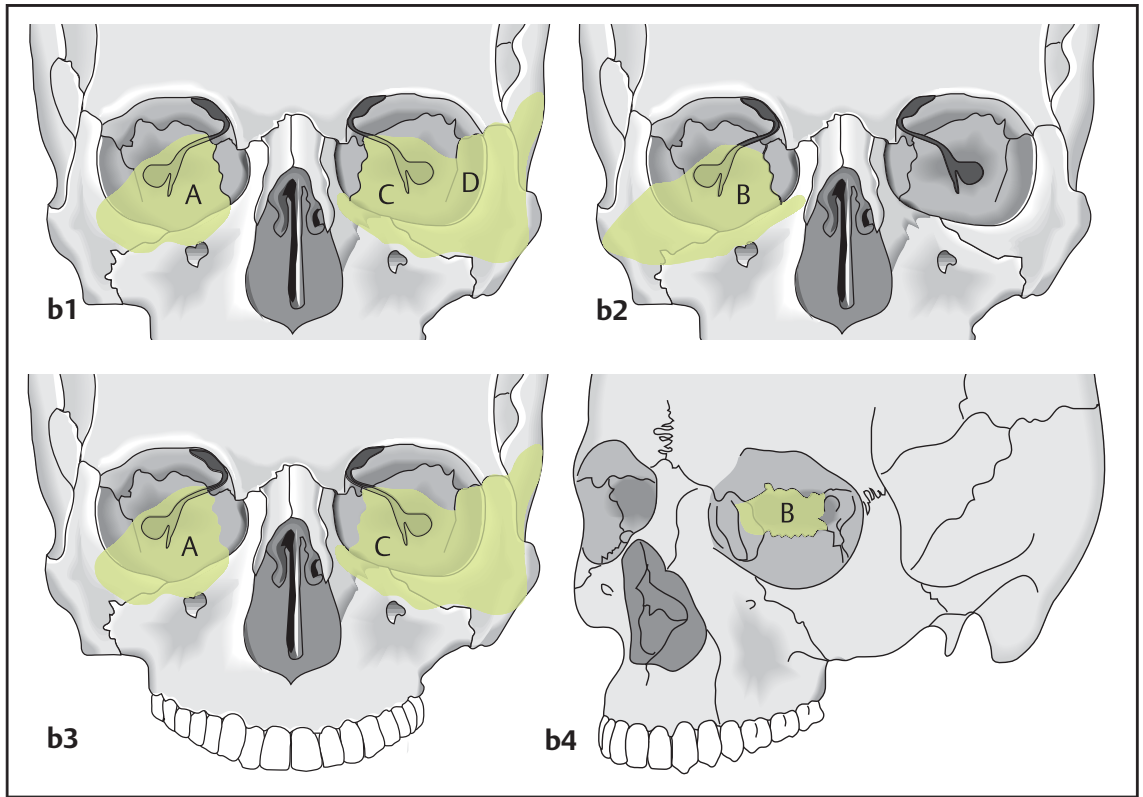

Abb.9b b1 Expositionsareale des Mittelgesichts/Orbita, die mit lokalen transkutanen Unterlidzugängen erreicht werden können (vgl. Abb.9a1). A subziliare Inzision; C infraorbitale Inzision; D subziliare Inzision mit lateraler Erweiterung. b2 B Expositionsareal des Mittelgesichts/Orbita nach subtarsaler bzw. „Mideyelid-Incision“. b3 Expositionsareale des Mittelgesichts/Orbita über transkonjunktivale Zugänge (vgl. Abb. 9d). A Untere Fornix-Conjunctivae-(= Bindehautwölbung)-Inzision; C untere Fornix-Inzision mit lateraler Kanthotomie (Swinging Eyelid). b4 Expositionsareale des Mittelgesichts/Orbita nach transkarunkulärem Zugang (B).

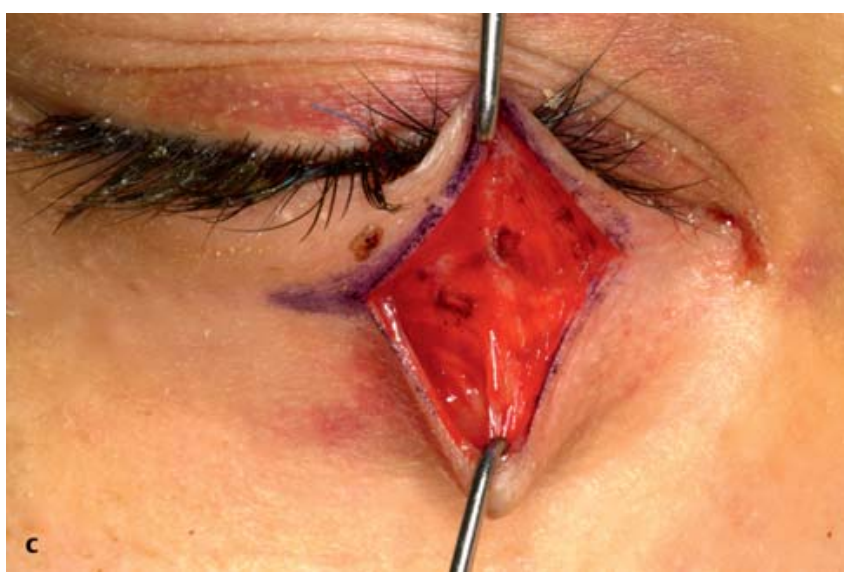

Abb.9c Mideyelid Incision und Darstellung des M. orbicularis oculi, der scharf durchtrennt wird.

\section{Operative Zugänge}

\section{Mid Eyelid Incision}

Dieser operative Zugang zum Boden der Orbita kann sehr schön in eine Hautfalte gelegt werden und ermöglicht eine gute Übersicht über den Boden und Anteile der medialen, sowie der lateralen Wand (Abb.9a). Die Inzision sollte nicht weiter als bis auf Höhe des lateralen Randes der Kornea geführt werden, damit die Narbe postoperativ annähernd unsichtbar bleibt. Nach erfolgter Hautinzision wird der M. orbicularis oculi scharf durchtrennt (Abb.9c) und suborbikulär, d.h. unter Erhalt des Septum orbitale auf der Rückseite des Muskels bis zum Infraorbitalrand präpariert. Es handelt sich um wie auch mit einem prä- oder transkarunkulären Zugang kombiniert werden, sodass große Anteile der lateralen wie auch der medialen Wand erreicht werden können. Bei Tendenz zur Narbenbildung im Bereich der Konjunktiva besteht das Risiko der Entwicklung eines postoperativen Entropiums.

\section{Blepharoplastikinzision lateral oder medial}

Die laterale obere Blepharoplastikinzision ist in erster Linie dazu geeignet, die Fissura zygomatico-sphenoidalis darzustellen und die Repositionsergebnisse im Bereich der Fissur und der lateralen Wand zu prüfen. Die Inzision erfolgt in einer Hautfalte und analog zum Vorgehen im Unterlid. Der laterale Kanthus kann dabei, je nach den topografischen Erfordernissen, abgelöst oder belassen werden (Abb.9e). Mithilfe der medialen oberen Blepharoplastikinzision können die oberen Anteile der medialen Wand unter Darstellung der A. ethmoidalis anterior und posterior eingesehen werden. Bei diesem Zugang muss die Trochlea, welche die Sehne des M. obliquus superior enthält, abgelöst werden. Die Trochlea reinseriert postoperativ spontan, funktionelle Probleme sind nicht $\mathrm{zu}$ erwarten. Berücksichtigt werden muss die anatomische Nähe des Zugangs zu den sensiblen Nn. supraorbitalis und supratrochlearis, sowie die enge Nachbarschaft der Austrittsstelle der A. ethmoidalis posterior mit dem N. opticus.

\section{Prä- oder transkarunkulärer Zugang}

Ausgedehnte, funktionell relevante Frakturen der medialen Wand können entweder über einen koronaren Zugang in Kombination mit einer Mid Eyelid Incision oder über einen prä- oder transkarunkulären Zugang eingesehen werden. Bei isolierter Fraktur der medialen Wand steht mit dem prä- oder transkarunkulären Zugang eine elegante lokale Alternative zur Verfügung [7,8]. Die Inzision der Konjunktiva erfolgt lateral der Plica semilunaris durch die Karunkel oder vor der Karunkel. Bei Erreichen der posterioren Begrenzung des Ductus lacrimalis wird das periorbitale Gewebe scharf durchtrennt und die mediale Wand subperiostal exponiert (Abb.9f). Begleitverletzungen wie ausgedehnte Frakturen des Sinus frontalis oder der Frontobasis erfordern einen koronaren Zugang. Diese Verletzungen müssen in enger Kooperation mit den mitbetreuenden Neurochirurgen behandelt werden. 

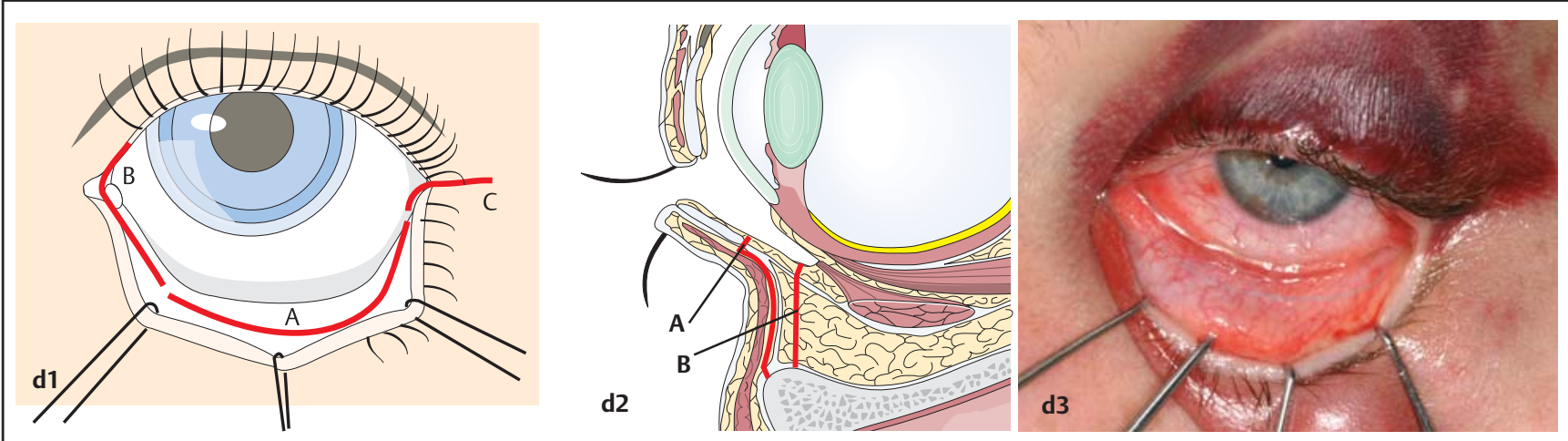

Abb.9d d1 Transkonjunktivale Unterlidzugänge im Schema: A untere Fornix-Conjunctivae(= Bindehautwölbung)-Inzision und Verwendung einer retroseptalen oder präseptalen Route zum Infraorbitalrand; B Trans-/retrokarunkulärer (= medial transkonjunktivaler) Zugang; C Untere Fornix-Inzision mit lateraler Hautschnitterweiterung (laterale Kanthotomie/Swinging Eyelid). d2 Passagewege von der unteren transkonjunktivalen Inzision zum Infraorbitalrand: präseptal (A) oder retroseptal (B). A präseptale Inzision, B retroseptale Inzision. d3 Transkonjunktivaler Zugang: Untere Fornix-Inzision - klinisches Beispiel.

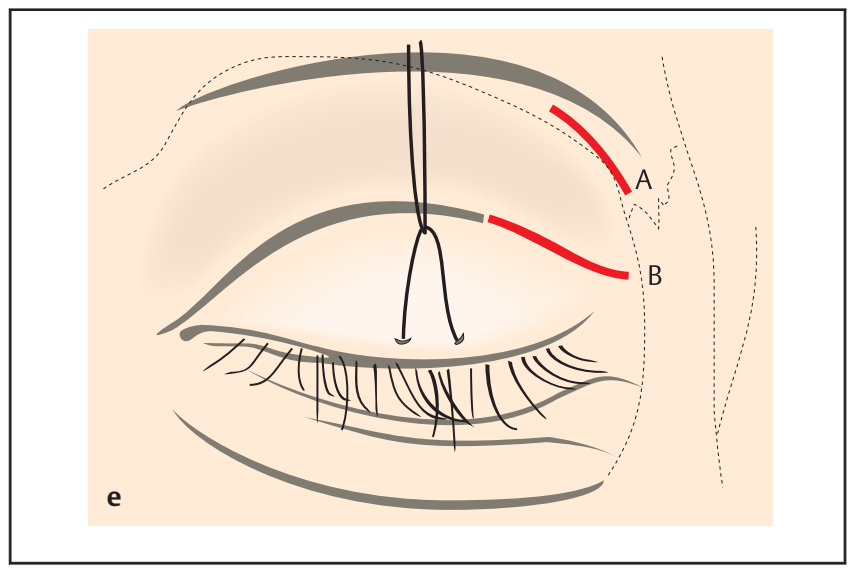

\section{Koronarer Zugang}

Der koronare Zugang oder Bügelschnitt ermöglicht die Exposition des gesamten oberen Anteils des Gesichtsschädels, der beiden Jochbögen, sowie der beiden Orbitae mit Ausnahme des Bodens und Anteilen der medialen Wand kaudal des medialen kanthalen Ligaments (Abb.9).
Abb.9e Transkutane Zugänge (Schnittführung) zur Crista frontozygomatica: A Laterale Braueninzision; B Obere laterale Blepharoplastik-Inzision.
In Kombination mit einer Mid Eyelid Incision können die Orbitae somit in ihrer gesamten Zirkumferenz dargestellt werden. Im Rahmen der Versorgung von panfazialen Frakturen ist dieser Zugang Voraussetzung zur korrekten 3-dimensionalen Rekonstruktion des Gesichtsschädels $[9,10]$. Muss aus Gründen der Frakturversorgung die Präparation bis auf den Jochbogen erfolgen, wird das tiefe Blatt der Temporalisfaszie auf Höhe des lateralen kanthalen Ligaments inzidiert. Die nachfolgende Präparation wird streng unterhalb der Faszie unter Schonung des $\mathrm{R}$. frontalis des $\mathrm{N}$. facialis geführt. Bei zu tiefem Eindringen in das temporale Fettgewebe besteht postoperativ die Gefahr der Atrophie des temporalen Fettkörpers (temporal hollowing) (Abb.9g5).

Ein großer Anteil der Frakturen der Orbitae kann unter der Verwendung von lokalen Zugängen versorgt werden. Bei zusätzlichen versorgungspflichtigen Frakturen des Sinus frontalis und bei komplexen Mittelgesichtsfrakturen (mehrfragmentärer Jochbeinkomplex, Stückfraktur des Jochbogens) können die korrekten Dimensionen des Gesichtsschädels (Höhe, Breite, Projektion) nur mithilfe eines koronaren Zugangs wiederhergestellt werden.

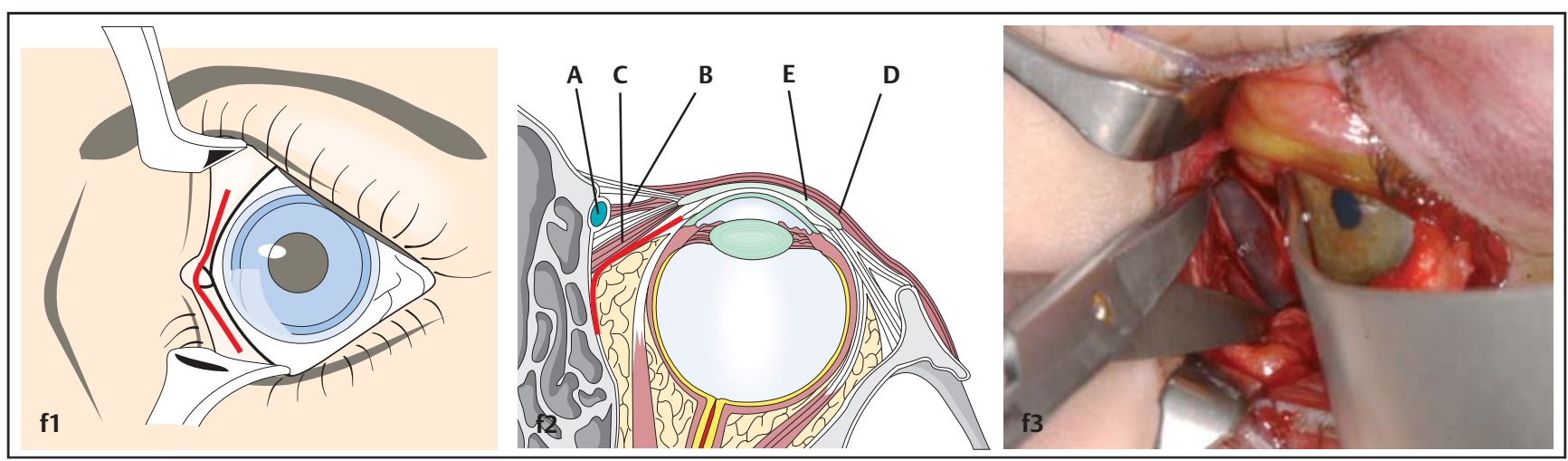

Abb.9f Transkarunkulärer Zugang: f1 Inzision in der semilunaren Konjunktivalfalte im Schema; f2 Präparationsroute entlang des Horner-Muskels zur medialen Orbitawand/dorsal des Tränenbeins in horizontaler Schnittebene: A Tränensack, B Jones Muskel, C Horner Muskel, D M. orbicularis oculi, E Tarsus; f3 Ansicht der medialen Orbitawand nach Eröffnung der Periorbita - klinisches Beispiel. Danach wird zum Schutz der Kornea die Konjunktiva temporär vernäht. 


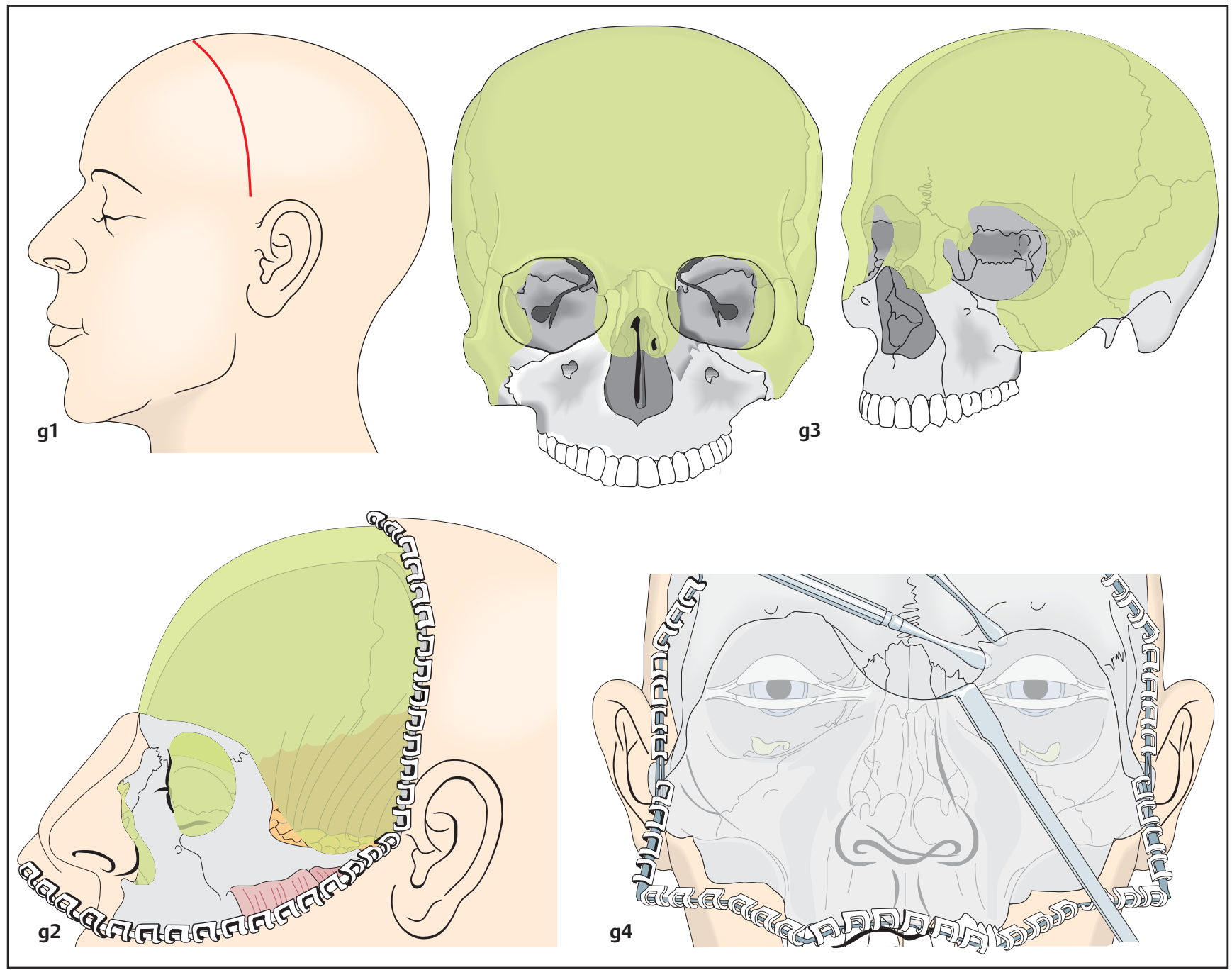

Abb. 9 g1 bis g4 $\mathbf{g 1}$ Koronarer Zugang - Schnittführung im Schema. g2 Koronarer Zugang - Entwicklung des Skalplappens über Schläfen und Stirnregion. g3 Expositionsareale im Hirn- und Gesichtsschädelbereich, die über einen koronaren Zugang erreicht werden können. g4 Darstellung des Skeletts im oberen Mittelgesichtsbereich und der oberen Orbita - Zirkumferenzen.

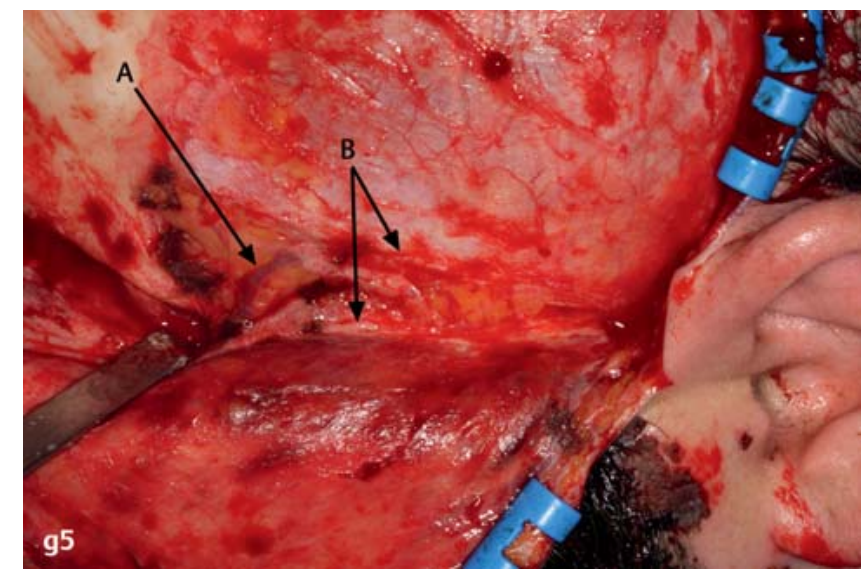

\section{Materialien zur Rekonstruktion}

Die anatomische Rekonstruktion der Orbitawände ist Voraussetzung für eine korrekte Position des Bulbus und ermög-
Abb.9g5 Koronarer Zugang: Anzeichnen der Sentinel-Vene $(A)$ im Bereich des tiefen Blattes der Fascia temporalis (B). Inzision der Fascia temporalis in diesem Bereich, dadurch Berücksichtigung des Verlaufs des R. frontalis des N. facialis. durch den Defekt nicht beeinflusst wird. Defekte bis zu einer Größe von $3 \mathrm{~cm}^{2}$ können grundsätzlich konservativ behandelt werden, unter der Voraussetzung dass $\leq 2 \mathrm{~mm}$ Enophthalmus vorliegt und keine Einklemmung von Muskel- oder periorbitalem Fettgewebe festgestellt werden kann [6]. Die Wahl des Rekonstruktionsmaterials richtet sich nach der Größe des Defekts und den zu erwartenden Belastungsverhältnissen. Basierend auf mathematischen Modellen ist es möglich, für einen definierten Defekt ein adäquates Rekonstruktionsmaterial auszuwählen [11]. Danach müssen Defekte der Kategorie A3 mit stabilen Materialien wie autologem Knochen oder Titangitter versorgt werden. licht die uneingeschränkte Motilität des Auges. Defekte des Orbitabodens kleiner als $1 \mathrm{~cm}^{2}$, die anterior des Äquators des Bulbus lokalisiert sind müssen nicht versorgt werden, da die Position des Bulbus 

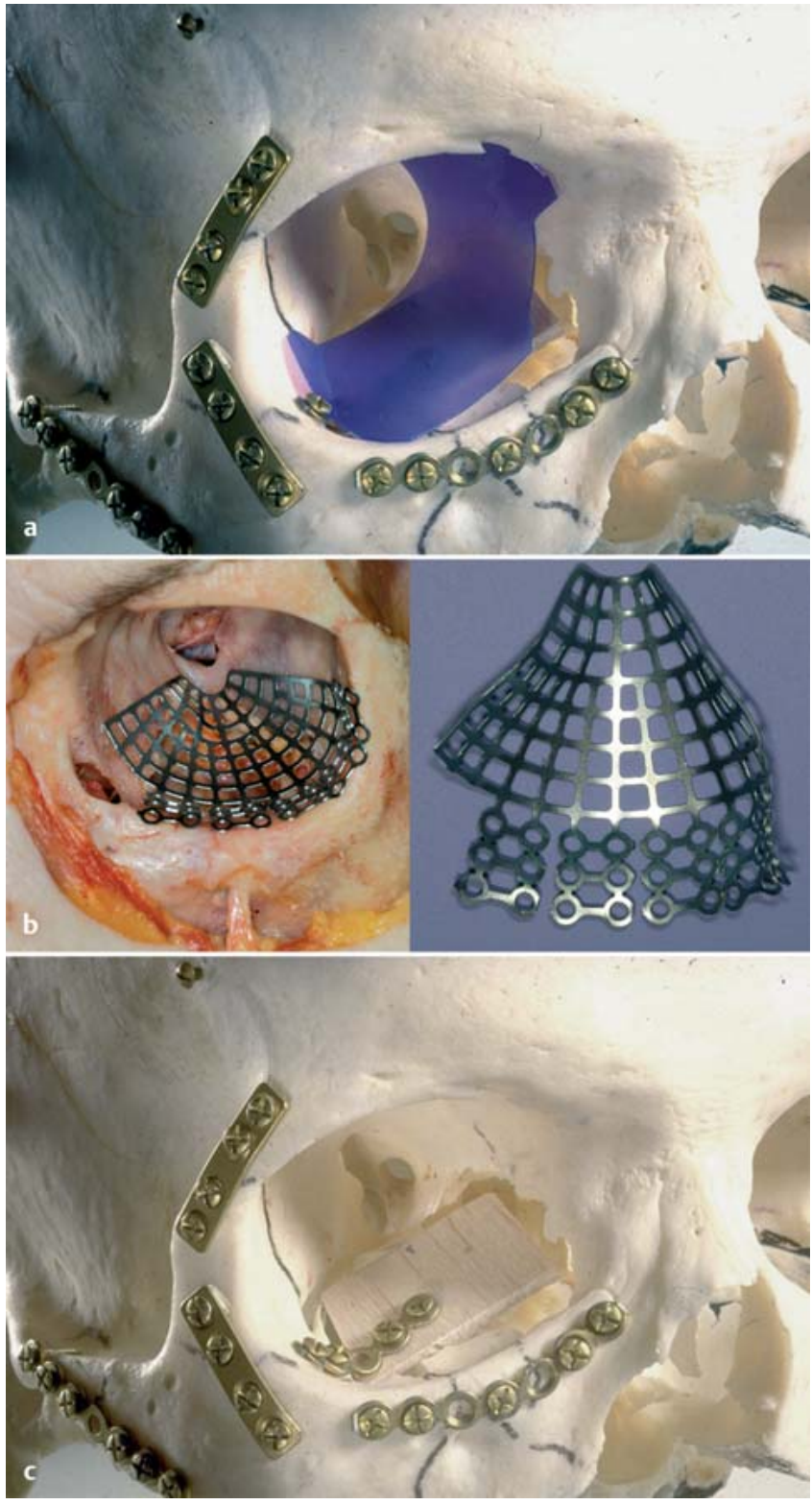

Abb. 10 a bis c a* PDS-Folie zur Schienung des periorbitalen Gewebes von medial bis zur lateralen Wand - Modell. b1 Rekonstruktion des Orbitabodens im Übergang zur medialen Orbitawandand (,key area“) mit einem Pie-shaped Titanium Mesh - Demonstration am anatomischen Präparat. b2 Konturgebung des Pie-shaped Titanium Mesh zum Ersatz von Orbitaboden im Übergang zur medialen Orbitawand. Zur Rekonstruktion der Key Area ist eine konvexe Wölbung im posteromedialen Anteil des Meshs notwendig. c* Rekonstruktion des Orbitabodens mit einem autologen Knochentransplantat vom Schädeldach - Modell (Balsaholz).

* Reproduced with permission from Orbital Fractures by Beat Hammer, ISBN 0-88937-139-3 and ISBN 3-8017-0785-2, pp. 56 and 57. (C) 1995 Hogrefe \& Huber Publishers Seattle Toronto Bern Göttingen

\section{Resorbierbare Materialien}

Resorbierbare Materialien wie PDS-Folien (Ethicon, Deutschland) sind geeignet, kleine Defekte bis zu einer Größe von $1-2 \mathrm{~cm}^{2}$ abzudecken. Mit Bezug auf die vorliegende Klassifikation ist dieses elastische Material für die Versorgung von Frakturen der Kategorien A1, A2, B1, B2 und C geeignet. Zusätzlich kann die PDS-Folie auch als Schienung und zum Schutz des periorbitalen Gewebes vor Einbringen von stabilen Rekonstruktionsmaterialien wie Titangitter verwendet werden (Abb. 10a).

\section{Nicht resorbierbare Kunststoffe (Polyethylene)}

Alternativ zur resorbierbaren PDS-Folie können auch nicht resorbierbare alloplastische Materialien wie Polyethylene $\left(\right.$ Medpor $^{\circledR}$, Stryker, Michigan, USA) eingesetzt werden. Diese Materialien bieten etwas mehr Stabilität, sind gleichzeitig aber weniger flexibel, weshalb die Insertion technisch anspruchsvoller ist. Grundsätzlich ist das Material für die gleichen Indikationen wie die PDS-Folie geeignet. Das Material ist in verschiedenen Dicken erhältlich und kann somit auch im Rahmen von sekundären Korrekturen (Enophthalmus) verwendet werden.

\section{Titangitter}

Titangitter sind die aktuell am häufigsten eingesetzten Materialien zur Rekonstruktion der Orbitawände. Bewährt haben sich insbesondere die sogenannten Pie-shaped orbital Meshes, die sich ausgezeichnet den individuellen anatomischen Verhältnissen anpassen lassen (Abb.10b). Aufgrund ihrer Stabilität können die Titangitter zur Versorgung sämtlicher Defekte der Orbitawände eingesetzt werden. Hinsichtlich postoperativer Komplikationen und Präzision des rekonstruktiven Ergebnisses lassen sich keine Unterschiede zu autologen Transplantaten nachweisen [12]. Bei ausgedehnten Defekten der Orbitawände gibt es die Möglichkeit der Verwendung von Preformed Titanium Meshes, die sich perfekt in die Anatomie der Orbita einfügen und nicht modifiziert werden müssen. Die Insertion dieser Implantate ist technisch sehr anspruchsvoll und erfordert eine genaue und vollständige operative Darstellung des zu deckenden Defekts.

\section{Autologe Transplantate}

Dank der hohen Präzision und ausgezeichneten klinischen Anwendbarkeit der Titangitter ist die Anwendung von autologen Transplantaten in den Hintergrund getreten. Autologe knöcherne Transplantate können einerseits aus dem Beckenkamm, anderseits aus der Schädelkalotte entnommen werden [13]. Nach der Entnahme müssen diese Transplantate entsprechend den Defekten individuell angepasst und am ortständigen Knochen fixiert werden (Abb.10 c). Bei den Transplantaten aus dem Beckenkamm handelt es sich embryologisch um enchondral gebildeten Knochen, reich an adulten Stammzellen, von denen sich die mesenchymalen Stammzellen potenziell in Osteoblasten differenzieren können. Die verfügbare Knochenmenge ist ausreichend. Wegen des großen Anteils an hämatologischen Stammzellen (Monozyten, Makrophagen, Osteoklasten) ist dieser Knochen infektresistent, neigt aber zu hoher Resorption im Rahmen der Integration des Knochens. Dies muss bei der Rekonstruktion der Orbitawände berücksichtigt werden. Aus der Tabula externa entnommener Knochen ist desmalen Ursprungs, reich an Kortikalis und entsprechend resorptionsresistent. Transplantate aus der Tabula externa sind bez. klinischem Handling und Beständigkeit im Langzeitverlauf die beste „autologe“ Alternative zu 


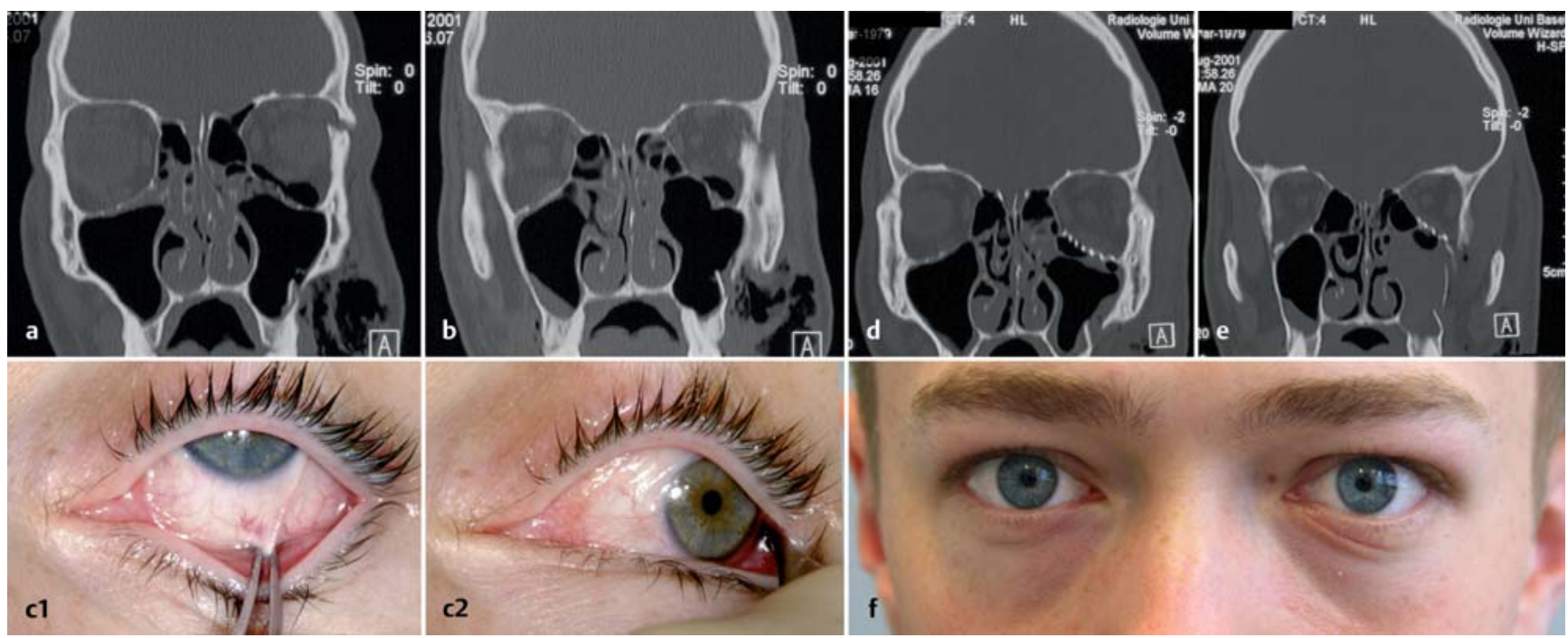

Abb. 11 a bis f a CT koronar: ausgeprägte Dislokation der Fraktur an der Crista fronto-zygomatica. b CT koronar, hinteres Drittel der Orbita: Lufteinschluss intraorbital und kleine Defektbildung im Bereich des Bodens der Orbita. c1 Passive Traktionskontrolle des Bulbus (Forced Duction Test) nach kranial. c2 Passive Traktionskontrolle des Bulbus nach lateral. d Erfolgte Reposition des Jochbeinkomplexes links, Rekonstruktion des Bodens der Orbita mit Titanmesh. e Korrekte Lage des Titangitters im hinteren Teil des Augenhöhlenbodens. f Dokumentation des Heilungsverlaufs: noch sichtbare Schnittführung ca. 3 Wochen postoperativ, symmetrische Bulbusstellung.

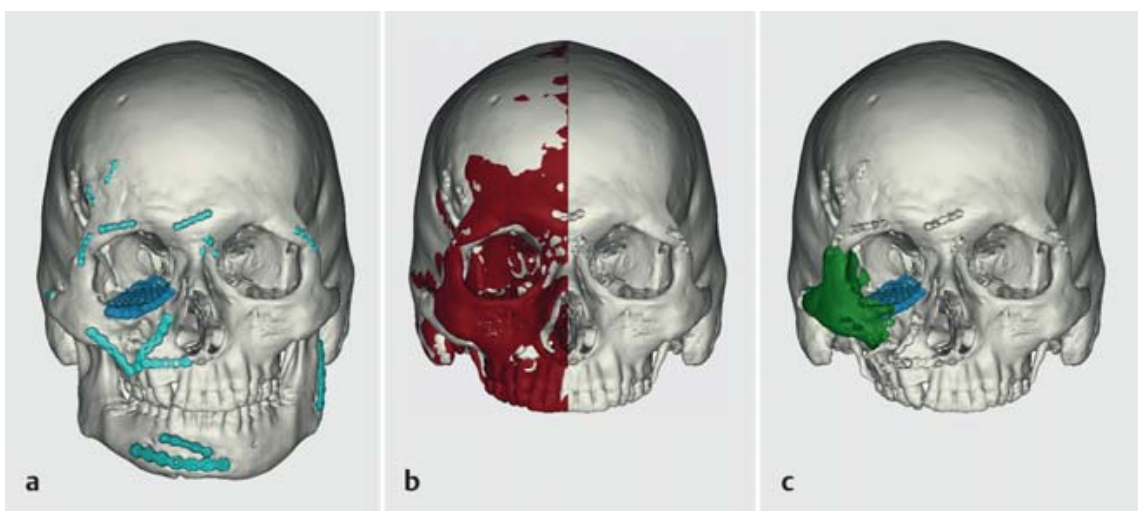

Abb.12a bis c a Zustand nach komplexer zentrolateraler Mittelgesichtsfraktur rechts. Blau: liegende Osteosynthese und Rekonstruktionsmaterial. Volumen der Orbita gegenüber der linken Seite erhöht. b Auf die rechte Seite gespiegelte linke Orbita. Rot: Volumenunterschiede zur angestammten Jochbeinregion. c Geplante Osteotomie des Jochbeinkomplexes links.

den Titangittern. Im Zusammenhang mit Tumoroperationen an der Orbita und nachfolgender adjuvanter Therapieformen (Radiotherapie, systemische Chemotherapie) sind Rekonstruktionen mit autologen Transplantaten zu empfehlen.

Frakturen $\geq$ Kategorie $A 3$ (Fraktur des Orbitabodens, der medialen Wand unter Erhalt der knöchernen Begrenzung der Fissura infraorbitalis) müssen mit stabiIen Materialien (Titanmesh) oder autologem Knochen versorgt werden.

\section{Operatives Vorgehen}

In der Abbildungssequenz 11 sind die einzelnen Schritte der Rekonstruktion und Osteosynthese bei einer Jochbein- fraktur mit Defektbildung im Bereich der Orbita zusammengefasst. Die präoperative CT-Diagnostik zeigt die Impression des Jochbeinkomplexes mit der Defektbildung im Bereiche des Orbitabodens (Abb.11 a, b). Entsprechend den Prinzipien des „outer facial frame“ wird zunächst der Jochbeinkomplex anatomisch korrekt reponiert und stabil verankert [9]. Im Anschluss daran erfolgt die Präparation der Defektgrenzen innerhalb der Orbita. In diesem Zusammenhang ist es notwendig, das in die Fissura orbitalis inferior einstrahlende Weichgewebe zu durchtrennen, damit insbesondere der dorsale Aspekt des Defekts klar dargestellt werden kann. Von medial und lateral präparierend kann so die dorsale Begrenzung des Defekts iden- tifiziert und freipräpariert werden. Die Positionierung des Implantats erfolgt unter Sicht und unter Kontrolle der Defektränder. Zum Schluss wird im sog. Forced Duction Test die passive Beweglichkeit des Bulbus in alle Richtungen sichergestellt (Abb.11 c). Postoperativ sollte wiederum eine CT-Untersuchung durchgeführt werden, um die korrekte Positionierung des Implantats zu überprüfen und dokumentieren (Abb. 11 d,e,f).

Vor Einsetzen eines Rekonstruktionsmaterials muss sichergestellt sein, dass die komplette Zirkumferenz der Fraktur dargestellt ist und das Material auf einer festen Unterlage abgestützt werden kann. Die postoperative CT-Untersuchung dokumentiert die korrekte Positionierung des Materials und schließt eine Einklemmung von periorbitalem Gewebe aus.

\section{Präoperative Planung und intraoperative Navigation}

Verletzungen des Gesichtsschädels sollten zeitnah operiert werden. Unter der Voraussetzung, dass die Versorgung innerhalb eines Zeitfensters von 5-7 Tagen erfolgen kann, ist nicht mit relevanten funktionellen Problemen $\mathrm{zu}$ rechnen [14]. Im Zusammenhang mit sekundären Korrekturen von in Fehlstellung verheilten knöchernen Verletzungen der Orbitae muss berücksichtigt werden, dass funktionelle Probleme auch nach Korrektur der knöchernen Orbitae persistieren können. Diese Probleme sind wahr- 


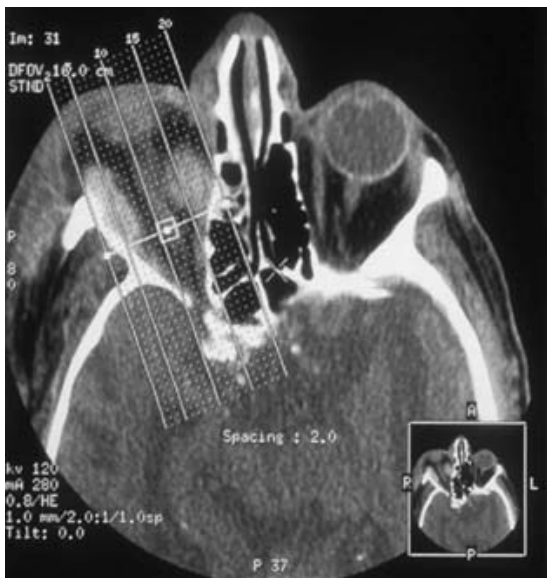

Abb. 13 Retrobulbäre Hämatome im axialen CT (Scout View mit Schnittebenen für multiplanare Darstellung.

scheinlich bedingt durch Atrophie und Narbenbildung innerhalb des periorbitalen Fettgewebes und der Bindegewebssepten. Bei der Planung und Durchführung von sekundären Korrekturen hat die virtuelle 3-dimensionale Planung in den letzten Jahren an Bedeutung gewonnen [15]. In einem ersten Schritt wird die nicht betroffene Orbita auf die betroffene Seite der Verletzung gespiegelt und farblich von der bestehenden Rekonstruktion unterschieden (Abb. 12a, b). Anschließend kann, wiederum farbcodiert, ein bestimmtes knöchernes Segment virtuell osteotomiert und in die vom Chirurgen gewünschte Position verschoben werden. Diese am Computer definierte Endposition (Abb. 12c) des osteotomierten Segments kann intraoperativ navigiert und kontrolliert werden [16].

\section{Postoperative Nachsorge und funktionelle Nachkontrollen}

Unmittelbar postoperativ müssen zu Beginn engmaschige Visuskontrollen durchgeführt werden. Bei Zunahme der Schmerzen postoperativ, bei kontinuierlicher Zunahme von Exophthalmus, sowie bei unterschiedlicher Wahrnehmung von Rottönen (Rotentsättigung im Vergleich zur Gegenseite) besteht klinisch Verdacht auf ein Retrobulbärhämatom, das zeitnah entlastet werden sollte (Abb. 13). Bei Auftreten einer Traktion auf den N. opticus kann es sehr rasch zum kompletten Visusverlust des betroffenen Auges kommen. Ein eingeschränk- tes Fusionsblickfeld postoperativ kann durchaus als Folge der postoperativen Schwellung gewertet werden unter der Voraussetzung, dass im Rahmen der postoperativen CT-Kontrolle keine mechanische Behinderung der Augenmotilität nachgewiesen werden konnte. Orthoptische Kontrollen sollten hingegen frühestens ca. 6 Wochen nach Rekonstruktion erfolgen. Eine Verbesserung des Fusionsblickfelds ist bis 12 Monate nach Rekonstruktion zu erwarten [5].

\section{Schlussfolgerung}

Knöcherne Verletzungen der Orbitae können nur mithilfe der Computertomografie (axiale und koronare Schichtung) korrekt diagnostiziert werden. Die Indikation zur operativen Versorgung erfolgt in der Gesamtschau von klinischen (Enophthalmus) und radiologischen Befunden. Bei den heute eingesetzten Rekonstruktionsmaterialien für die Versorgung von relevanten Frakturen $\left(\geq 3 \mathrm{mc}^{2}\right)$ haben sich im Wesentlichen Titangitter durchgesetzt. Bei korrekter Indikationsstellung und zeitnaher operativer Versorgung ist die Prognose von Verletzungen der Orbitae ausgezeichnet.

\section{Literatur}

${ }^{1}$ Koornneef L. Orbital septa: anatomy and function. Ophthalmology 1979; 6: 876-880

${ }^{2}$ Ellis E 3rd, El-Attar A, Moos KF. An analysis of 2067 cases of zygomatico-orbital fractures. J Oral Maxillofac Surg 1985; 43: 417-428

3 Bowers JF. The management of blow-out fracture of the orbital floor. Surv Ophthalmol 1964; 40: 237-243

${ }^{4}$ Manson PN, Iliff N. Management of blow-out fractures of the orbital floor II. Early repair for selected injuries. Surv Ophthalmol 1991; 35: 280-292

${ }^{5}$ Jaquiéry C, Aeppli C, Cornelius $P$ et al. Reconstruction of orbital wall defects: critical review of 72 patients. Int J Oral Maxillofac Surg 2007; 36: 193-199

${ }^{6}$ Kunz C, Sigron G, Jaquiéry C. Functional outcome after non-surgical management of orbital fractures - the bias of decision-making according to size of defect: critical review of 48 patients. Br J Oral Maxillofac Surg 2013; 51: 486-492

${ }^{7}$ Kris SM. The precaruncular approach to the medial orbit. Arch Facial Plast Surg 2003; 5: 483-487

8 Shorr N, Baylis HI, Goldberg RA et al. Transcaruncular approach to the medial orbit and orbital apex. Ophthalmology 2000; 107: 14591463

9 Gruss JS, Bubak PJ, Egbert MKA. Craniofacial fractures. An algorithm to optimize results. Clin Plast Surg 1992; 19: 195-206
${ }^{10}$ Gruss JS, Whelan MF, Rand RP et al. Lessons learnt from the management of 1500 complex facial fractures. Ann Acad Med Singapore 1999; 28: 677-686

11 Van Leeuwen AC, Ong SH, Vissink A et al. Reconstruction of orbital wall defects: recommendations based on a mathematical model. Exp Eye Res 2012; 97: 10-18

12 Ellis E 3rd, Tan Y. Assessment of internal orbital reconstructions for pure blow-out fractures: cranial bone grafts versus titanium mesh. J Oral Maxillofac Surg 2003; 61: 442453

13 Tessier P, Kawamoto H, Posnick J et al. Taking calvarial grafts, either split in situ or splitting of the parietal bone flap ex vivo - tools and techniques: A 9650-case experience in craniofacial and maxillofacial surgery. Plast Reconstr Surg 2005; 116: 54S-71S; discussion 92S-94S

14 Girotto JA, MacKenzie E, Fowler C et al. Longterm physical impairment and functional outcomes after complex facial fractures. Plast Reconstr Surg 2001; 108: 312-327

15 Gellrich NC, Schramm A, Hammer B et al. Computer-assisted secondary reconstruction of unilateral posttraumatic orbital deformity. Plast Reconstr Surg 2002; 110: 1417-1429

${ }^{16}$ Juergens $P$, Klug C, Krol Z et al. Navigationguided harvesting of autologous iliac crest graft for mandibular reconstruction. J Oral Maxillofac Surg 2011; 69: 2915-2923

\section{Priv.-Doz. Dr. med. Dr. med. dent} Claude Jaquiéry

Oberarzt

Priv.-Doz. Dr. med. Dr. med. dent. Christoph Leiggener

Oberarzt

Priv.-Doz. Dr. med. Dr. med. dent. Christoph Kunz

stellvertretender Chefarzt

Klinik für Mund-, Kiefer- und

Gesichtschirurgie

Universitätsspital

Spitalstrasse 21

4031 Basel

Schweiz

cjaquiery@uhbs.ch

Prof. Dr. med. Dr. med. dent. Carl-Peter Cornelius

Oberarzt der Klinik

Klinik und Poliklinik für Mund-, Kiefer- und Gesichtschirurgie Klinikum der Universität München Ludwig-Maximilians-Universität München Lindwurmstr. 2a

80337 München 